\title{
Smart Bin for Waste Managemen Using CPaaS Clouds \& IoT
}

\author{
Sameen Firdous*, Zahid Altaf, Gazamfar Nabi, Riaz Ahmed Khan \\ Department of Computer Science \& Engineering, Islamic University of Science \& Technology, Awantipora, \\ Jammu \& Kashmir
}

Received: 16 February 2020; Accepted: 25 March 2020; Published: 08 April 2020

\begin{abstract}
The incorporation of technology in waste management of smart cities will immensely contribute to the reduction of cost and complexities of current waste management systems by improving their efficiency, health safety, and productivity. It will also help to lessen the negative impact of waste management activities on the environment, thus contributing to the smart city scenario. Present designs are not refined enough to accomplish a liberal, generous and viable waste organization segment.Various solutions have been offered for implementing smart collection, transportation, and disposal of waste, yet efficient waste management has not been achieved. . It is basic to have a sharp technique for directing and coordinating waste and also the garbage level should be educated in-time when to be taken, and likewise, all the accomplices should know that what kind of waste in what sum needs to be collected.To ensure such efficient collection and management of waste, we propose a "Smart Bin for Waste Management System using Communication Platform as a Service Clouds \& Internet of Things (IoT)”. In this prototype system, a unique ID is assigned to each garbage bin. The bins are equipped with an embedded system to measure the garbage level and other indicators such as temperature and tilt in the garbage bin. These sensors detect different events such as the bin getting filled up to its maximum level or tilting beyond a certain threshold and the system sends an alert message to the municipal authorities or central monitoring station using SMS, E-mail, \& Voice-call. It also provides route optimization to the pickup vehicle, responsible for garbage collection in the concerned area.
\end{abstract}

Index Terms: Texas Instruments CC3200, HC-SR04 Ultra-Sonic Sensor, DFR0028 Tilt Sensor, Energia, Temboo and Twilio CPaaS Clouds.

(C) 2020 Published by MECS Publisher. Selection and/or peer review under responsibility of the Research Association of Mode rn Education and Computer Science

\footnotetext{
* Corresponding author.

E-mail address:
} 


\section{Introduction}

Overall enthusiasm for smart cities has increased, encouraged by the need to discover compelling solutions for the significant challenges expected in the coming years. Among these, inculcating innovations in waste management is a major challenge [1]. Waste collection is a basic city administration service. An adequate opportunity exists worldwide for smart technology to build proficiency and improve the nature of waste collection administrations. At present, most metropolitan waste collection operations centre on emptying bins or containers as per pre-defined plans. This is highly inefficient, with half-full containers being emptied and poor utilization of city resources plus unnecessary fuel utilization. Over the past decade, Internet of things (IoT) has and continues to reform society in various spaces like human services, smart homes, well-planned urban areas, etc. [2].

The interconnection via the Internet of computing devices installed in everyday objects, empowering them to send and get information, is commonly termed as the Internet of Things (IoT). The IoT is an idea wherein surrounding things, with the help of wired and remote frameworks are connected without client intervention. In IoT, the objects convey and exchange information to give smart organizations to customers. The IoT has been able to increase extensive academic interests because of the ongoing advances in smart-phones, equipped with different communication modules, network technologies (Wi-Fi, LTE), and sensors. In academia, industry, and government as considerable IoT application fields, waste management has also become a symbolic concern. There are various factors, like an inefficient and indiscriminate discharge of waste, inefficient waste management strategies, and absence of waste management and dumping systems that have led to gained significant costs for waste disposal and genuine natural issues. Therefore, IoT based-Smart bin is one particular solution. In past various IoT based waste management projects are being designed expecting to give a powerful framework for effective waste collection. However they lacked the desired efficacy and innovation. Many works didn't utilize constant data of waste assortment. Although a few methodologies utilized propelled plan and directing by means of information and communication technology calculations and algorithms. Some relevant works have used such modules and components in their framework that has decreased their performance as well as effectiveness and at the same time increased their complexity. Thus, there was a need for a better and advanced innovation with IoT that uses less complicated and comprehensive hardware and provides an interface that can be used for making the whole waste management process efficient and dynamic. In our proposed framework, real-time information about the garbage bins status can be available via Internet. Management duties can be carried out aptly to curtail operational costs by optimizing the quantity and deployment of effective technology for garbage collection [3]. The proposed work delineates how the Smart Bin arrangement enables authorities to recognize waste management issues progressively. We present a plan of cloud framework for association of waste assortment procedure and applications for drivers and agencies. Gathering information and data progressively can help authorities from squandering and thus protect assets and be cost effective.

\section{Related Work}

In the past, since human population density and level of misuse of standard resources were less, the quantities of solid waste produced were also much lower than what they are today. Since the human population has sharply risen, so has the waste. To keep the environment clean and healthy, there is a need for proper waste disposal. Inappropriate waste disposal prompts pollution, health problems, different risks and other negative impacts on the environment. Pollution seriously affects the developing and populated urban communities as it contains contaminants which result in instability, disorder, and damage to the ecosystem. 
Thus, proper waste removal and management are necessary to improve the health and wellbeing of the city's population. In this context, we evaluated several reports and briefly summarized them below:

Glouche et al. (2013) [4] proposed a Radio Frequency Identification (RFID) based waste management system. In this system, tags are assigned to each dustbin, which help in monitoring waste in the dustbin. The authors emphasize on the recycling of waste present in the dustbins, depending upon the tag assigned. Hong et al. (2014) [5] proposed an IoT based framework, in which dustbins were battery based and exchange data through wireless networks. This system is server based. Servers and routers are required for the analysis of information.

A. Medvedev et al. (2015) [6] proposed a system, "Decision Support System" (DSS) for garbage collection. The system is based on data sharing between garbage-truck drivers in real-time to perform garbage collection and which helps in dynamic route optimization. Aazam et al. (2016) [7] proposed a framework called” Cloud-based smart waste management for smart cities.” This framework emphasizes on making all the stakeholders aware that what kind of garbage is getting filled in garbage bins and at what particular time. This framework needs continuous monitoring of data. S.Saranya et al. (2017) [8] proposed a system called "IoT Based Garbage Monitoring System”. In this system, CC3200 micro-controller (MCU) with on-board Wi-Fi is used to monitor the level of the trash present in the dustbin. This system also gives information about the trash level present in the waste-bin with the help of web server. The system provides smart solution for monitoring garbage yet it lacks efficiency and productivity, as the system provides no smart solution for garbage clearance. More-over, the system is Global System for Mobile Communication (GSM) based which is contradictory to the use of on-chip Wi-Fi connectivity MCU, "CC3200”.

S. Mirchandani et al. (2017) [9] proposed a smart monitoring and clearance system by using a smart bin technology wherein the garbage bins had GPS module \& a GSM system installed within them which was interfaced with a micro-controller. There was a force sensor that calculated the weight of the bin, once the bin hits full it sends its location by the data obtained from the GPS module with the help of the GSM system to the authorities. The authorities are equipped with an Android app that gets a notification once the bin is full \& a collection agent is sent to collect the garbage. Each bin also had a unique RFID to store the dustbin data \& its location. Al-Masri et al. (2018) [10] proposed a server-less framework that detects the sources of violations which could lead to proper waste management. The authors emphasize on the public awareness about the subject.

After studying these and few other related frameworks, we found that while they are beneficial for waste management but they have certain drawbacks and limitations. For example, the greater part of them use GSM/GPRS module which is not a great choice for an IoT based System as for every unit or system, there's a necessity of SIM(subscriber character module or supporter ID module). In pretty much every framework, when a condition, for example, 'container is filled', is met an alert signal or message is generated. An alert signal or message must be generated only if the condition endures or persists for some time, avoiding a false alert.

\section{Problem Statement}

Current waste management system employed in Jammu \& Kashmir and in most of the country is the traditional system of collection and management of waste. Traditional waste management systems are resource-intensive, inefficient and expensive [11, 12]. Currently, waste is collected at certain intervals by garbage trucks. The garbage trunks have to roam all around the cities to collect the filled bins. At some places the bins get filled up much faster than others and are already overflowing by the time of waste collection whereas at other places the bins are half empty at the time of collection. This leads to improper management, more fuel consumption, man-power, increase in traffic jams, and also poses hygiene risks. 
According to experts, if there is a smart waste collection system, then the manpower required will be $90 \%$ less than that of the traditional waste collection system.

Considering the problems in traditional waste collection system, we propose a smart bin for waste management with the following objectives:

1. To design a bin with garbage level checking and an SMS, call \& E-mail based alert system.

2. To design a framework that can provide location and path to a filled bin.

3. To design a framework that can detect the tilt of the bin.

4. To design a framework that can detect open lid of the bin.

5. To design a framework that can detect fire in the bin.

6. To design a framework that provides Wi-Fi-Service around the bin.

\section{Proposed System}

After a thorough literature survey, we developed a solution that had a new dimension altogether. Our proposal (Fig.1) aims at managing the garbage that is collected in bins in a smart way. Our proposal enables us to monitor the garbage level through ultrasonic sensors and when full, using cloud technology, alerts are sent to the authorities for its proper management, collection and disposal. In this way, with this framework, the public and the government authority can be assured that prudent steps are taken to fight the waste collection issues.

This proposal 'Smart Bin for Waste Management using CPaaS Clouds \& IoT' is an endeavor to design a framework that will help keep the urban communities clean. The framework helps monitor the garbage bins and level of solid waste gathered in the bins and bothersome changes in their inclination/orientation through incorporated CPaaS clouds (Temboo \& Twilio). To distinguish the trash level and contrast it with the garbage bins depth, ultrasonic sensors are used. In this framework, ultrasonic sensors are utilized for detecting open lid of the dustbin by analyzing the distance between the lid and the side of the bin. Tilt sensors are utilized in the framework to identify tilts in particular directions. The framework uses the CC3200 launch-pad for sending information. The framework is controlled by a $5 \mathrm{~V}$ battery. The framework additionally gives location information and path of each bin which could be displayed to municipal vehicles liable for maintaining and clearing the bins in their particular regions and the central administrator of the municipality.

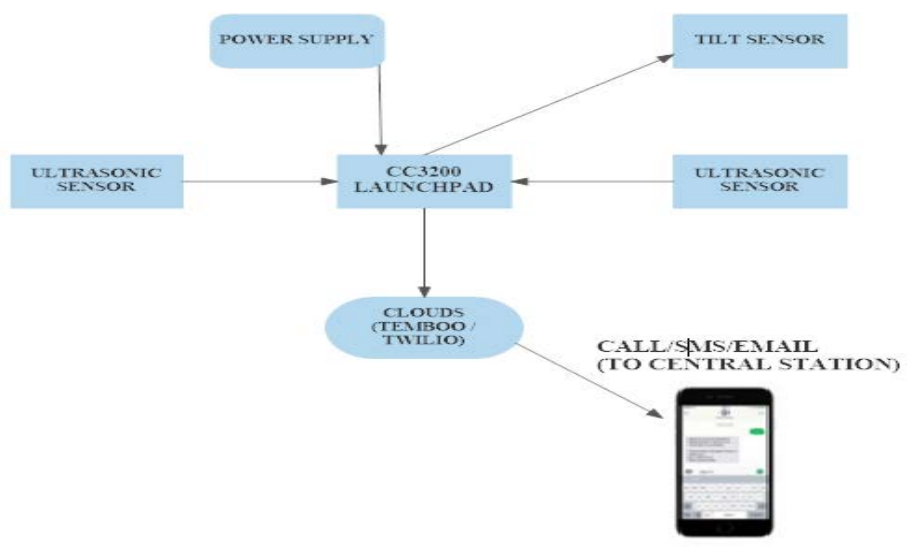

Fig.1. Proposed System 


\section{FLOW OF CONTROL/SIGNAL:-}

For this proposal, we are utilizing TI CC3200 Launch-pad MCU, which is the main part of the system, temperature Sensor (TMP006) for fire alert, ultrasonic sensor(HC-SR04) for garbage level and open lid check , tilt Sensor (DFR0028) for detecting the tilt and clouds (Temboo and Twilio) for transmission of information (Fig 1). The flow of signal is as follows:-

1. Firstly, ultrasonic sensors are utilized to check levels, tilt sensors are utilized to identify the tilt and the onboard temperature sensor for distinguishing temperature changes when the ideal conditions are met, a trigger occurs.

2. After the trigger occurs, an alert signal is sent to CC3200 MCU via the interfacing wires, which connects to Temboo Cloud.

3. Temboo Cloud at that point utilizing our Wi-Fi's credentials and Twilio Library sends us alerts (SMS, Call, and E-mail) by means of Twilio Cloud (Fig.2). These alerts help in knowing the location of the bin as well as path to the bin (Fig.3).

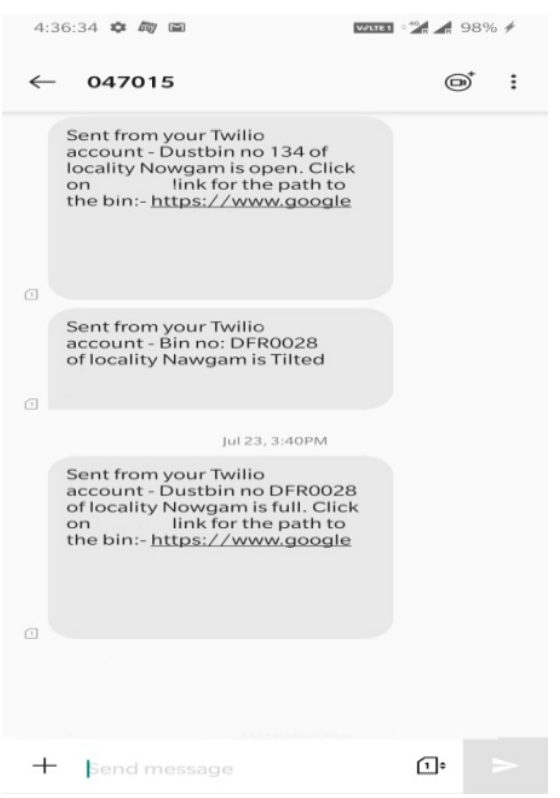

Fig.2. SMS/Call/Email alert

The following are the results obtained after the successful performance testing of the work:

- Accurate Garbage level detection (Fig 4)

- Wireless transmission of real time information to the authorities

- Cloud integration: SMS / Call / e-mail based alert system

- Open lid and tilt alert (Fig 2)

- Exact Location and path to the filled bin (Fig 3) 


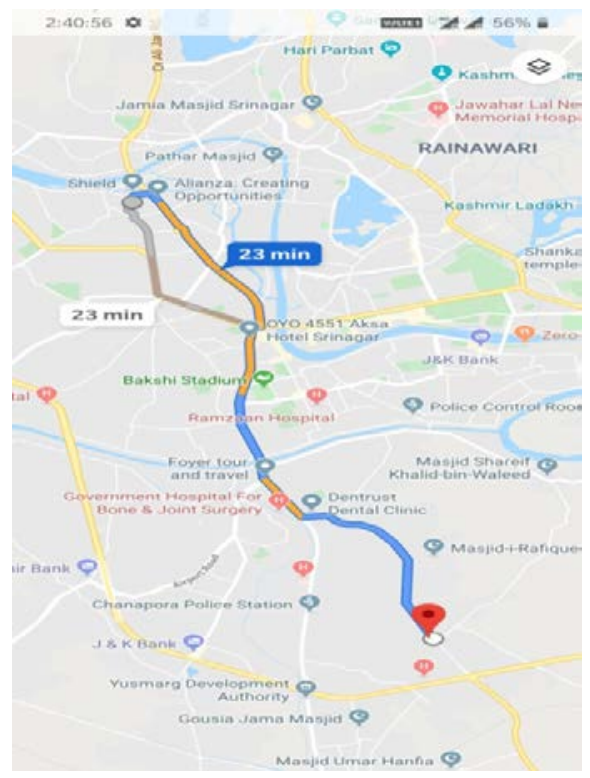

Fig.3. Illustrating path to the bin

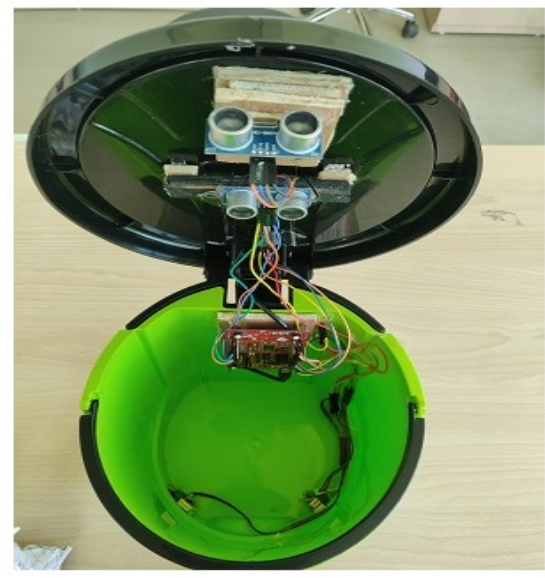

EMPTY SMART BIN

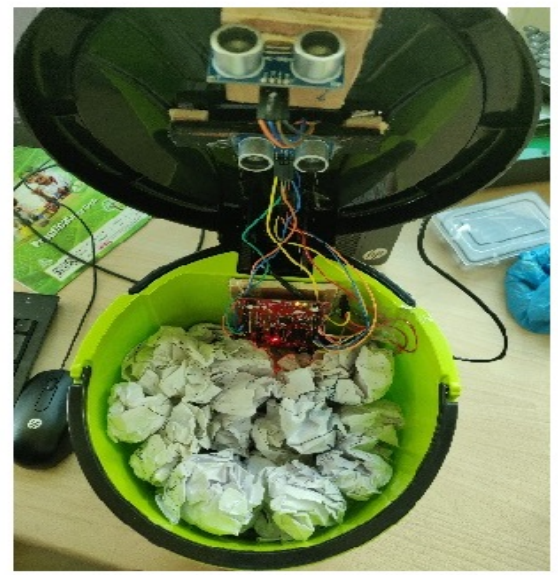

FULL SMART BIN

Fig.4. Smart Bin 


\section{Implementation}

For the implementation of proposed framework following hardware and software have been used: -

\subsection{TI CC3200 LaunchpadXL:-}

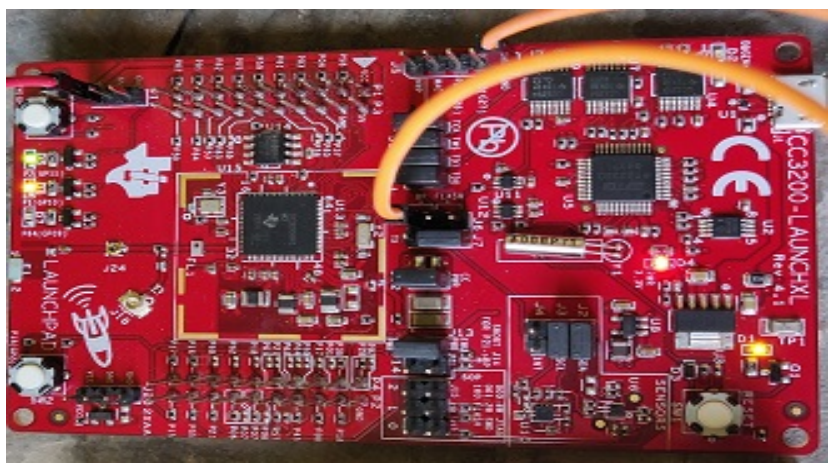

Fig.5. TI CC3200 Launch-pad-XL

CC3200 Launch-pad XL (Fig. 5) is a single-chip micro-controller unit with on-board Wi-Fi. It is first Wi-Fi CERTIFIED single chip micro-controller unit. It is a wireless micro-controller that integrates micro-controller, ARM-Cortex-M4 for the development of application with a single IC. It is designed particularly for IoT applications and no prior Wi-Fi tech. experience is needed for the faster development.

CC3200 Launch-pad XL is a single-chip micro-controller unit (MCU) with on-board Wi-Fi connectivity. It is first Wi-Fi CERTIFIED single chip MCU. It is a wireless MCU that integrates ARM-Cortex-M4 MCU, for the development of application with a single IC. It is designed particularly for IoT applications and no advance Wi-Fi tech. knowledge is needed for the faster development [13, 14].

In this framework, as the core component, CC3200 MCU has been programmed to use data from various sensors, including temperature sensor on-board and interfaced with cloud platforms for alert delivery.

\subsection{ULTRASONIC SENSOR HC-SR04:-}

HC-SR04 (Fig. 6) is a four pin module with pins VCC, Echo, Ground and Trigger. It is a quite popular sensor, used for measuring distance and sensing objects. The sensor consists of two projects in front that act as Ultrasonic transmitter and Receiver.

Working formula: $\quad \mathrm{k}=\mathrm{v} \times \mathrm{t}$

Where $\mathrm{k}=$ distance, $\mathrm{v}=$ speed, $\mathrm{t}=$ time

An ultrasonic wave is transmitted by the Ultrasonic transmitter, when this wave hits any material it gets reflected back and is observed by Ultrasonic receiver [15].

FLOW CHART:- The ultrasonic sensor has been configured to send signal to the CC3200 MCU indicating imminent garbage overflow whenever its distance from the solid waste in the bin is measured less than $7 \mathrm{~cm}$. The MCU, upon reception of the signal, alerts the Temboo cloud platform. (Fig. 7) 


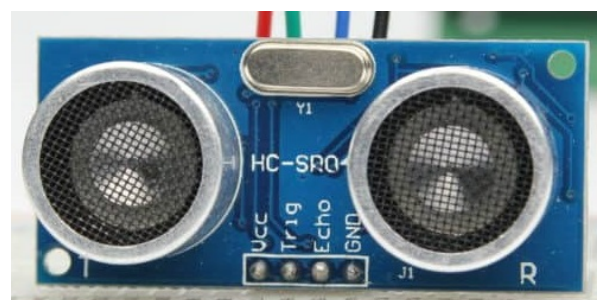

Fig.6. HC-SR04

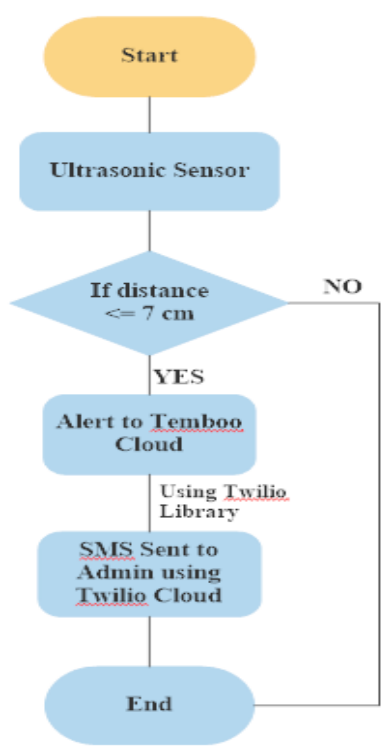

Fig.7. HC - SR04 Flow Chart

In the framework, the function of second ultrasonic sensor is to alert open lid of the bin. This sensor is configured to work in a way such that a signal is sent to the CC3200 MCU if the distance between this sensor and the wall of the bin it faces exceeds $15 \mathrm{~cm}$.

\subsection{DIGITAL TILT SENSOR DFR0028:-}

A digital interfaced and mercury based tilt sensor (Fig.8). Its module is based on various mercury switches. The supply voltage is between $3.3 \mathrm{~V}$ to $5 \mathrm{~V}$ and size is $22 \mathrm{x} 30 \mathrm{~mm}$ [16].

FLOW CHART: Upon getting tilted, the tilt sensor sends signal to the CC3200 MCU indicating change in orientation of the bin. The MCU, being interfaced to the Temboo cloud, automatically alerts tilted condition of the bin. (Fig. 9) 


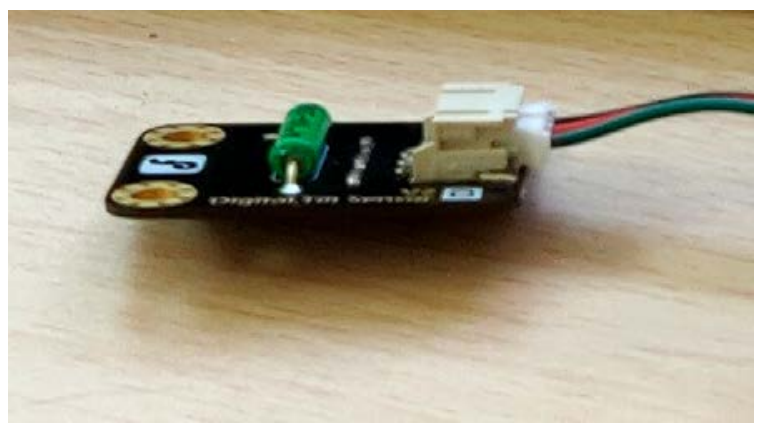

Fig.8. Tilt Sensor (DFR-0028)

FLOW CHART: Upon getting tilted, the tilt sensor sends signal to the CC3200 MCU indicating change in orientation of the bin. The MCU, being interfaced to the Temboo cloud, automatically alerts tilted condition of the bin. (Fig. 9)

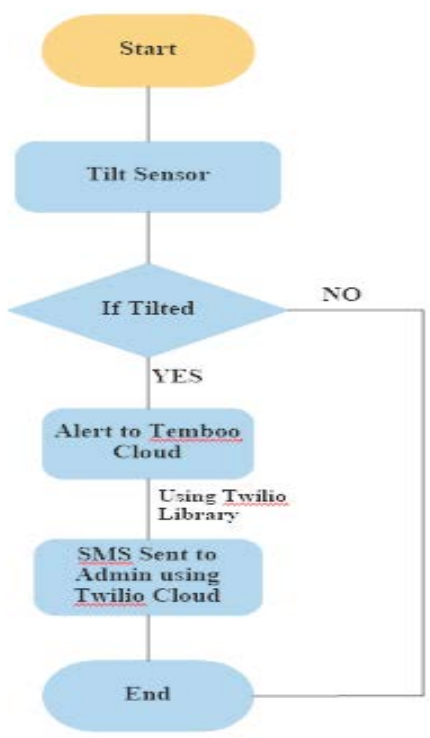

Fig.9. Flow chart of DFR0028

\subsection{ENERGIA Integrated Development Environment(IDE):-}

Energia (Fig.10) is a community-driven and an open-source Integrated Development Environment \& software. It provides a coding platform, APIs and libraries that help in writing code for a micro-controller [17].

In this proposal, Energia has been used to push/flash code written in C to the MCU CC3200 platform. This includes input handling, conditional statements and cloud interfacing. 
The implementation of our proposed framework led us to make a system that automates waste collection and transport process. The system detected filled bins and provided optimized route for collection of bins. The system also detected the tilt, open lid of the bin, fire in the bin and provided respective SMS/CALL/Email alerts.

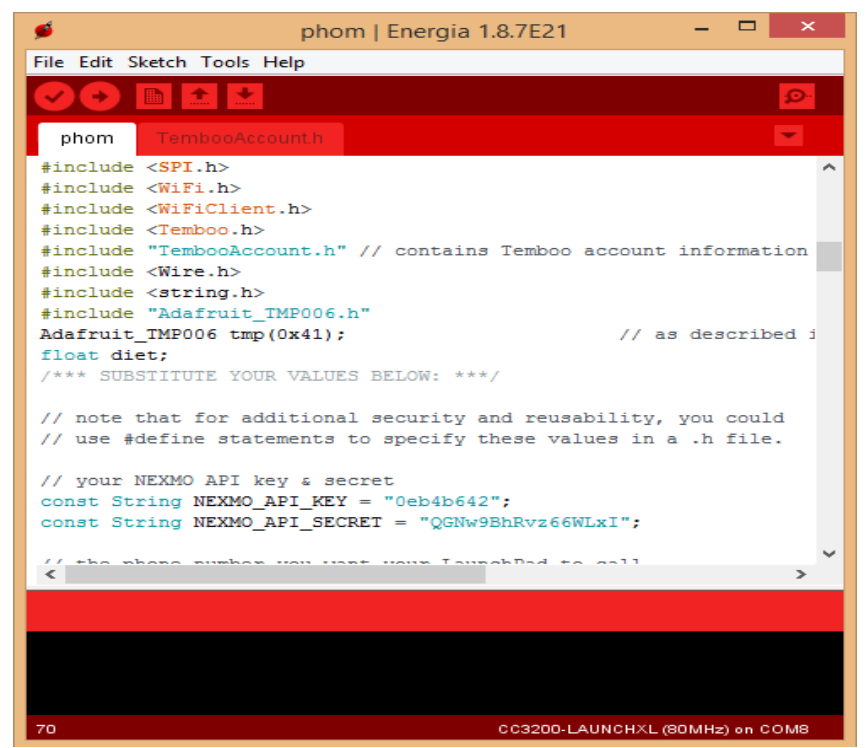

Fig.10. ENERGIA IDE

\subsection{ADVANTAGES OF PROPOSED FRAMEWORK: -}

1. Only filled waste-bins are collected by municipal-corporation trucks/workers.

2. Deployment of waste-bins based on actual needs.

3. Cost diminution and resource optimization.

4. Better environmental quality.

5. No trash odour.

6. Route optimization.

7. Fire alert.

8. False alarm check.

9. Wi-Fi facility

\section{Conclusion, Summary \& Future Scope}

With the expanding populace and changes in the way of life, management of waste has become a challenge for the authorities. It has become critical to have a vigorous method for dealing with the waste such that the entire procedure is smooth and removal of waste is cost effective and gainful. To achieve the same we have designed a smart bin for waste collection/management system using IoT that helps in checking the fill level of bins whether they are filled or not. In addition, we have interfaced the system with tilt and temperature sensors. The bins can be managed efficiently as the SMS, Call, \& Email-based alert system informs the concerned authorities in time and a decision can be taken duly. By implementing this proposed system or framework, cost diminution, resource optimization and effective utilization of smart bins can be ensured. 
More-over, this system indirectly contributes to reducing traffic in the city. In major cities, garbage collection vehicles go round each region every day, more than once or twice relying upon the number of inhabitants of the particular area and sometimes these bins might not be filled. Our System will check the status of every bin and inform the status of bin (that is filled) in real-time to the concerned authority so that necessary action can be taken.

Future work and extension in this system may include the implementation of operational analytics and data analysis [18].This encompasses a software suite for accessing, managing and collecting the data [19, 20].

\section{Competing Interests}

The authors have no competing interests. The research did not receive any specific grant from funding agencies in the public, commercial, or non-profit sectors.

\section{ACKNOWLEDGEMENTS}

The authors wish to thank the faculty of Centre for Development of Advanced Computing (C-DAC) Mohali, India, and Dr. Riaz Ahmed Khan, Assistant Professor of Department of Computer Science \& Engineering, Islamic University of Science \& Technology, for their support to the research.

\section{References}

[1] Mahajan, S. A., Kokane, A., Shewale, A., Shinde, M., \&Ingale, S. (2017). Smart Waste Management System using IoT. International Journal of Advanced Engineering Research and Science, 4(4).

[2] Nirde, K., Mulay, P. S., \&Chaskar, U. M. (2017, June). IoT based solid waste management system for smart city. In 2017 International Conference on Intelligent Computing and Control Systems (ICICCS) (pp. 666-669). IEEE.

[3] Piyare, R. (2013). Internet of things: ubiquitous home control and monitoring system using android based smart phone. International journal of Internet of Things, 2(1), 5-11.

[4] Glouche, Y., \& Couderc, P. (2013, June). A smart waste management with self-describing objects.

[5] Hong, I., Park, S., Lee, B., Lee, J., Jeong, D., \& Park, S. (2014). IoT-based smart garbage system for efficient food waste management. The Scientific World Journal, 2014.

[6] Medvedev, A., Fedchenkov, P., Zaslavsky, A., Anagnostopoulos, T., \&Khoruzhnikov, S. (2015). Waste management as an IoT-enabled service in smart cities. In Internet of Things, Smart Spaces, and Next Generation Networks and Systems (pp. 104-115). Springer, Cham.

[7] Aazam, M., St-Hilaire, M., Lung, C. H., \& Lambadaris, I. (2016, October). Cloud-based smart waste management for smart cities. In 2016 IEEE 21st International Workshop on Computer Aided Modelling and Design of Communication Links and Networks (CAMAD) (pp. 188-193). IEEE.

[8] Saranya, S., Yuvapriya, K., \&Sangeetha, P. (2017). IOT Based Garbage Monitoring System For Smart City Using CC3200”. International Journal of Future Innovative Science And Engineering Research, 3(1).

[9] Mirchandani, S., Wadhwa, S., Wadhwa, P., \& Joseph, R. (2017, December). IoT enabled dustbins. In 2017 International Conference on Big Data, IoT and Data Science (BID) (pp. 73-76). IEEE.

[10] Al-Masri, E., Diabate, I., Jain, R., Lam, M. H. L., \& Nathala, S. R. (2018, October). A Serverless IoT Architecture for Smart Waste Management Systems. In 2018 IEEE International Conference on Industrial Internet (ICII) (pp. 179-180). IEEE.

[11] Dash, Nivedita (23 June 2017). "New list of 30 smart cities is out. Did your city make the cut?” India Today. Archived from the original on 23 June 2017. Retrieved 23 June 2017. 
[12] Shyam, G. K., Manvi, S. S., \& Bharti, P. (2017, February). Smart waste management using Internet-of-Things (IoT). In 2017 2nd international conference on computing and communications technologies (ICCCT) (pp. 199-203). IEEE.

[13] Ti.com, 'CC3200 SimpleLink Wi-Fi ${ }^{\circledR}$ and Internet-of-Things solution, a Single-Chip Wireless MCU', 2015. [Online]. Available: http://www.ti.com/product/CC3200. [Accessed: 20-Jul-2019].

[14] Ti.com, 'SimpleLink Wi-Fi CC3200 LaunchPad', 2015. [Online]. Available: http://www.ti.com/tool/CC3200-LAUNCHXL. [Accessed: 20-Jul-2019].

[15] XeranRai,'Ultrasonic Sensor', 2019. [Online].Available:https://www.academia.edu/40147810/ ULTRASONIC_SENSOR.[Accessed:01-Aug-2019].

[16] Dfrobot.com, 'Digital Tilt Sensor SKU DFR0028', 2019. [Online].Available: https://wiki.dfrobot.com/Digital_Tilt_Sensor_SKU_DFR0028.[Accessed: 01-Aug-2019].

[17] Ti.com, 'ENERGIA', 2015. [Online]. Available:http://www.ti.com/tool/ENERGIA. [Accessed: 01-Aug-2019].

[18] Neil Sequeira,'IoT Applications in Waste Management'. 2019. [Online]. Available: https://www.iotforall.com/iot-applications-waste-management. [Accessed: 01-Aug-2019]

[19] Christina Jung, 'IoT and Smart City trends boost smart waste collection market'.2017. [Online].Available:https://www.greenbiz.com/article/iot-and-smart-city-trends-boost-smart-waste-collec tion-market. [Accessed: 01-Aug-2019]

[20] Khan, Z., Anjum, A., \&Kiani, S. L. (2013, December). Cloud based big data analytics for smart future cities. In 2013 IEEE/ACM 6th International Conference on Utility and Cloud Computing (pp. 381-386). IEEE.

\section{Author's Profile}

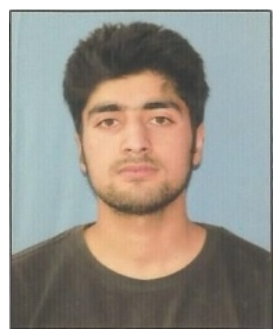

Sameen Firdous, Department of Computer Science \& Engineering, Islamic University of Science \& Technology, Awantipora, Jammu \& Kashmir- 192122.

How to cite this paper: Sameen Firdous, Zahid Altaf, Gazamfar Nabi, Riaz Ahmed Khan. "Smart Bin for Waste Managemen Using CPaaS Clouds \& IoT ", International Journal of Education and Management Engineering(IJEME), Vol.10, No.2, pp.38-49, 2020.DOI: 10.5815/ijeme.2020.02.05 